\title{
Is There a Role for an Evolutionary Genetics Based Rational Health Policy In Global Biomedical, Health and Economic Policies?
}

\section{Ferez S Nallaseth ${ }^{*}$}

Principal Investigator \& Consultant, Life Sciences Institute of New Jersey, New Jersey, USA; Member, Complex Biological Systems Alliance, Boston, MA, USA and Rutgers Cancer Institute of New Jersey, New Brunswick, NJ 08093, USA.

*Corresponding author: Ferez S Nallaseth, Principal Investigator \& Consultant, Life Sciences Institute of New Jersey, New Jersey, USA, Tel: 6462835163 , 908431 5069; E-mail: ferez.nallaseth@gmail.com, ferez.nallaseth@cbsaimtt.com

Rec date: June 27, 2014; Acc date: June 28, 2014; Pub date: June 30, 2014

Copyright: (c) 2014 Nallaseth FS. This is an open-access article distributed under the terms of the Creative Commons Attribution License, which permits unrestricted use, distribution, and reproduction in any medium, provided the original author and source are credited.

\section{Article}

The Biomedical Sciences Community has placed an exclusive emphasis on retrospective intervention(s) in managing pathological consequences, in thousands of diseases which cost hundreds of trillions of dollars per annum. In the treatment of some intractable and ghastly diseases, which are far more dehumanizing than any Death Camp, such as cancers and neurodegeneration(s) these approaches are largely ineffective. These diseases can only be successfully treated by preemption! Breaking the cycle perpetuating this paradigm and prospectively preempting disease causing mutations that escape the networks of genes maintaining chromosome and genome biology in mammalian development and homeostasis requires a combinatorial approach including complementary contemporary and classical methods with the recruitment of an evolutionary genetics approach for systematizing and controlling this network. This enterprise is predicated on a trans generational and international commitment through a framework of Institute(s) of Functional Mammalian Chromosome And Genome Biology funded by a variation of President Barack Obama's budgetary rescue of the Detroit Motor Industry. The need is fulfilled by training, more and not fewer Ph.D. scientists with some re-directed to this goal, whose contributions will gradually diminish rates of disease allowing the re-allocation of hundreds of trillions of dollars spent each year from perpetuating the management of consequences of diseases to productive applications in Global Health and Economic Policy. The time scales for realization of these benefits of necessity, must be measured against the scale of many current ineffective therapies, and extended into perpetuity. Although based on other similar enterprises, returns on investment are likely to be accelerated once critical thresholds of data and knowledge are attained. As in anything meaningful that has ever been achieved in civilization, e.g. exploration of Space these commitments must be enduring. And so if humans never launched a Sputnik they could never build a Space Station!

Right now, and more than at any time before, there is a strikingly desperate global need - and the Biomedical Scientific community seems to be abdicating its responsibilities in addressing it! What is this urgent need? Before addressing this issue it must be pointed out that I have been pressured in sustained and innumerable ways to desist from commenting on things that are way beyond my pay grade (easily done)! However, there is such a thing as the First Amendment in the Constitution of the United States of America. Beyond the US and certainly the narrow needs of the Sciences, we all know of striking examples from History that compel our actions and expressions. Yes, there is an important place in it, for the likes of Drs., Albert Einstein and Fred Sanger who have as much a hand in the ongoing revolution or renaissance in the Sciences as anyone at any time in History! As well as for the likes of, Isaac Newton, Charles Darwin and Gregor Mendel! But there is also a unique place in it, not just for Scientists but for those many others who resisted and in doing so transformed Humankind leaving behind a better and less repressive world! They include the likes of Drs.: Andrei Sakharov and Yelena Bonner, Nikolai Vavilov, Robert Oppenheimer, Barbara McClintock, Marie Curie, Stephen Hawking, Martin Luther King as well as JBS Haldane, President Nelson Mandela, Daw Aung San Suu Kyi, Mahatma Gandhi, President Abraham Lincoln and all those under-privileged and non-titled ordinary people such as Medgar Evers and Freedom Riders who became resistance fighters without the entitlements and benefits of academic shelter and yet either step or stepped out of the pages of History! So I will follow their example and repeat myself - what is this urgent need?

It is outlined as structural flaws in the Biomedical Sciences and authored by Scientists, each of whose various contributions (scientific and otherwise) come from the very Pinnacle of Science, including Nobel Laureates, in two singularly important reports, one from the United Kingdom and the other from the United States [1,2]. In essence they identified the pressures of inadequate funding (albeit recognized as generous within the limited goals of Science that they perceived as adequate) as being responsible for having spawned mindless competition, negative non-productive behaviors, corrupted peer review processes, loss of scientific creativity, the practice of 'safe science' with all its stifling constraints, pernicious and insidious nepotism leading to unsustainable growth of record numbers of scientists produced by mentors and the current system. These are largely on target - but essentially only a small part of a much larger problem! I subscribe to the Academic Dictum that all knowledge is good! But when confronted with these overwhelming limitations of funding and resources, the systematic exclusion of serendipity e.g. as in the discovery of Penicillin by Dr. Alexander Fleming [3] with its massive returns for Humankind and its replacement by a Myopic Hypothesis Driven system of evaluation (which is based on the assumption that all knowledge is known!) jeopardizing the fundamental mission of the Biomedical Sciences community, the sobriquet of 'Boutique Science' is thoroughly applicable!

Before continuing a qualifier is necessary! I know well, that as a Scientist, I will never make anywhere near as large a contribution as each of the authors of these two reports (ignoring the reasons - for now!), but have to respectfully disagree with some of their conclusions. Whereas the concerns and solutions in these reports have some currency, in essence they miss the 'Forrest for the Trees' - which is completely in keeping with the current paradigm into which the Biomedical Sciences community is locked. I have no doubt as to my insignificance but in all fairness have to offer two qualifying examples. Firstly, at one time the likes of Drs., Barbara McClintock [4] and Marie 
Curie [5] met similar responses, and without elaboration, for similar social reasons. And secondly a good question is a good question regardless of its source! By extension, I know that the question that we have posed could neither be meaningfully addressed, by at least one Nobel Laureate (NL) author of the above reports, nor by several other Biomedical Scientists, ranking from NLs to Graduate Students.

What is this problem, which when addressed, will bring the current crises in the Biomedical Sciences under an umbrella of solutions? Before even beginning the process of answering this question, the perspectives have to be shifted from the constraints of Academia, the 'Immediate Here and Now of Local Science' as well as the Health Industry, such as the largely 'domestic' preoccupations of tenure, rank, funding, peer review, publications, teaching loads, revenue, patents, etc..! They have to be broadened to include the larger Historical perspectives and responsibilities of Bench Science which led to the spawning of the, Industrial Revolution, Information Age, Space Age, Green Revolution and many other revolutions that are underway. A process, which along with that of Democracy was responsible for nothing less than the Personal Freedoms of those fortunate few who had access to its benefits. And like Democracy, the scientific process does have flaws, despite which both have yielded the means for Human kind to do nothing short of wresting its personal freedoms from essentially ruthless and capricious feudal social systems! For some of the most striking of these flaws in Science read Dr. Hawkings's description [6] of one of his most ruthless predecessors who occupied the same Lucasian Chair of Physics at Cambridge University that he now holds and who came from the very Pinnacle of Science. He has successors in our age!

However, to return to the immediate and key problem confronting science and society on a global scale, one that arises from remaining locked in a paradigm that deprives us of credibility - and so of adequate funding and resources! It is our inability to make any significant impact on particularly intractable, ghastly and dehumanizing diseases like many cancers, neuropathological (Strokes, Alzheimer's and Parkinson's) and developmental (Down's, Spina Bifida) diseases [7-9].The reason is simple - the only cure for these diseases is prospective preemption! This inability to preempt them stems largely from ad hoc responses to individual diseases, dismissal of classical methods as passé and over-dependence on contemporary technologies almost as a panacea rather than the judicious and indispensable combined application of these methods in elucidating the universal underlying and causal problem i.e. disease causing mutations (used in the generic sense and recognized as necessary if insufficient)! The Biomedical Sciences community is locked into a paradigm which perpetuates the retrospective management of consequences of disease states (e.g. pathologies, symptoms) rather than prospectively preempting the escape of the causative mutations from the networks of genes maintaining the genome in mammalian homeostasis and development. Evolutionary Genetics and its near infinite supply of variants, when coupled with contemporary methods would readily allow both the systematization AND CONTROL of these networks and thus the preemption of disease causing mutations. Pre-emption is the ONLY way that some intractable diseases can be cured! \{(a) More information and methods are listed in references [10-14] and (b) contemporary methods such as OMICS, CRISPR/Cas9 excision,, Biomarkers, Stem Cell Therapy, etc.. although remarkably powerful and relevant for now, do belong to retrospective interventions and will do absolutely nothing to forestall new mutations with their burgeoning human and economic costs. CRISPR/Cas9 can neither excise mutations in multi copy sequences e.g. mitochondrial DNA in cardiomyopathies nor make relevant excisions of primary mutations that have become largely irrelevant after unleashing irreversible pathologies driven by secondary cascades e.g. mutations, dys-regulated miRNA, epitomics, expression, etc... As in some cancers.)\}. Nothing will replace the combinatorial approach of classical and contemporary methods in preempting disease states! Why does this classical genetics approach still remain an indispensable complement to contemporary approaches? Because it allows the identification of mechanisms and pathways that insert disease causing mutations into sequences resident at their native loci, with their specific chromatin and interphase nuclear conformations after being selected over evolutionary time spans, thus escaping from the regulatory genetics of the mechanisms evolved to maintain particular loci (with some overlaps and coregulation)! Diseases like cancer are caused by the serendipitous subversion of these mechanisms operating at specific loci - and neither by some brilliant mutagenic screen nor by directed mechanisms with potentially toxic drug/auxotrophic marker selections either operating on or controlled by e.g. transgenes and knockouts. Nothing will replace the systematization and control of the networks of genes maintaining the genome in mammalian homeostasis and development!

What are the broad technical and scientific landmarks in the development of Institutes leading to the systematization and control of the networks maintaining chromosomes and the genome in mammalian homeostasis and development including screens for mutations? They are: (1) documentation of high frequency mutations (up to a million times higher than neutral mutation frequencies), e.g. at Y chromosomal and some SNP loci exponentially increasing the likelihood of detection [10-14] (2) the presence of a high diversity of well characterized cis and trans acting genomic, chromosomal and interphase nuclear reporter targets, [10-14], (3) documentation of high numbers of known interspecific combinations of genomes inducing specific classes of dysfunctions of chromosome and genome biology e.g. in natural hybrid zones (NHZ) of mice [10-14], (4) well established and evolutionarily conserved, co-segregating or overlapping or serendipitously co-acting genes regulating maintenance of target reporter loci and chromosomes such as the 875 genes known to constitute the DNA Integrity Network in yeast [10-14], (5) known contemporary and classical tools such as OMICS, mouse-human chromosomal synteny, orthology, gene and cell ablation to complement evolutionary genetics by e.g. up or down regulating expression of locus specific maintenance and repair genes as required [10-14] (6) known thresholds of data leading to histograms of controlling and target genes/loci in this network with consequent transitions from incremental to logarithmic rates of advancement e.g. the OMICS and Stem cell revolutions [10-14] (7) separate budgets for the proposed Institute(s) from those of major funding agencies relatively easily financed by variations on President Obama's rescue of e.g. Detroit's Auto Industry with a fraction of the budgets of all State and Federal Departments, Pharmaceutical, Petroleum, Automobile and other Polluting Industries and Sin Taxes for a budget that is the equivalent of that of NASA at $\$ 20$ billion per annum and (8) other natural organizational and administrative concerns in setting up and maintaining an enterprise of this dimension.

What would be the financial benefit of 'A Proposal for A Rational Health Policy'? All Federal sources of funding for basic research totaled $\$ 156$ billion in 2009 (Appendix table 4-29) [15]. The highly conservative costs of $\sim 10$ major diseases (e.g. cancers, neuropathologies $(\mathrm{AD}, \mathrm{PD}$, etc...) and Cardiac conditions (which are multi-factorial) were conservatively estimated at $\$ 47$ trillion per year [16]. A minimum of 4000 and possibly more than 10,000 monogenic 
mutations have been reliably quantified as causing disease states, while the other 4 genetic causes such as aneuploidies, translocations, multifactorial and mitochondrial mutations $[7,8,9]$ are still being quantified. Therefore there is a striking disparity between funding for preemption of mutational causes (e.g. suggested $\sim 20$ billion dollars/ annum), the current funding of all sciences by the Federal Government (156 billion dollars) and the expenditures of hundreds of trillions of dollars for managing the pathological consequences of disease made in perpetuity! Should the Biomedical Sciences Community succeed in making inroads into solving this unprecedented problem, which is the single largest problem confronting Science, Health and Economic Policy on a Global Scale, the public perceptions of the field being the equivalent of a 'Boutique Enterprise' would be dissipated (eventually) and the 'Systemic Flaws' identified in one of those position papers would follow in short order! $[1,2,10-14,17,18]$. Transcending the reservations of the global community can only be effected by bridging this chasm through systematizing and controlling the networks of genes from which disease causing mutations escape, thus preempting disease states. This in turn would require the building of a (network of?) Institute(s) of Functional Mammalian Chromosome and Genome Biology! $[17,18]$.It would also require subsuming the narrow 'domestic priorities' of Academic and Industrial Scientific communities which would thus be firmly placed on the way to solving the particular professional malaise by which they are currently consumed.

By eventually breaking out of the current paradigm into which the Biomedical Sciences community is locked, including our acquiescence in the hapless acceptance of ghastly diseases to which Humans are subjected as inevitable, we not only relieve the perpetual pressure and wastage of hundreds of trillions of dollars, but of more importance, we address an issue of Human Costs that has dogged Humankind over Evolutionary time spans! These are the thoroughly dehumanizing and horrible consequences of many intractable disease states exacted in ways that the most ruthlessly and pathologically brutal of Death Camp Guards would not have the remotest ken to inflict!

\section{References}

1. Brenner S (2014) Interview By Dzeng, E. How Academia and Publishing are Destroying Scientific Innovation: A Conversation with Sydney Brenner, King's Review Blog/Magazine.

2. Alberts B, Kirschner MW, Tilghman S, Varmus (2014) Rescuing US biomedical research from its systemic flaws 111, PNAS
3. Sir Alexander Fleming.

4. Barbara McClintock (2014) The Barbara McClintock Papers, Profiles in Science, National Library of Medicine.

5. Marie and Pierre Curie (1996) Marie and Pierre Curie and the Discovery of Polonium and Radium, Nobelprize.org.

6. Hawking S A (1988) Brief History of Time, Bantam Books, Random House, ISBN 0-553-10953-7 to ISBN 0-553-38016-8.

7. Gene Mutations and Disease (2005) National Cancer Institute.

8. Genetics Home Reference (2014) National Library of Medicine, National Institutes of Health.

9. Genes and Human Disease (2014) World Health Organization.

10. Nallaseth FS (1992) Sequence Instability and Functional Inactivation of Murine Y chromosomes can occur on a Specific Genetic Background, Mol Biol Evol 9: 331.

11. Nallaseth FS (2007) Genetic Regulation of the Structure of Murine Y chromosomes As a Model For Systematizing Locus and Chromosome Specific Maintenance of Genomes Developing Mammals, Abstract, International Symposium On Chromosomes to Genome, Centre for Cellular and Molecular Biology, Hyderabad, India .

12. Nallaseth FS, Guo ZS (2011) Structural destabilization of Y chromosomes in interspecific backcrosses is consistent with variations in epigenetic modifications, Transgenerational effects, Poster 280 C, Genome Stability and DNA Repair, Genetics Society of America, Model Organisms to Human Biology- Mouse Genetics, Washington, D.C.

13. Nallaseth FS, et al (2012) Dissecting developmentally and physiologically relevant regulation of mammalian chromosome biology with murine interspecific back crosses, Y chromosomes, unstable inverted repeat (IR) Sry loci and HJ replication restart complexes, Abstract 109, Genome Stability and DNA Repair, Genetics Society of America, Model Organisms to Human Biology- Cancer Genetics, Washington, D.C.

14. Nallaseth FS, et al (2012) Dissecting physiologically and developmentally relevant genetic regulation of mammalian chromosome biology with murine interspecific backcrosses, $\mathrm{Y}$ chromosomes, unstable inverted repeat (IR) Sry loci, sex reversal phenotypes, viral Oris and HJ-replication restart complexes: A synopsis, Program/Abstract \#431, Society for Developmental Biology, 71st Annual Meeting In association with the Sociedad Española de Biología del Desarrollo Montreal, Canada.

15. Appendix Table 4-29 (2012) Chapter 4, Science and Engineering Trends, National Science Foundation.

16. The Global Economic Burden of Non-communicable Diseases (2011) a report by the World Economic Forum and the Harvard School of Public Health.

17. Nallaseth FS (2013) A Proposal for a Rational Health Policy.

18. Nallaseth FS (2014) Website Introduction . 